\title{
CONTRACTILE PROPERTIES OF THE MUSCLES OF MASTICATION OF RHESUS MONKEYS (MACACA MULATTA) FOLLOWING INCREASE IN MUSCLE LENGTH
}

\author{
J. A. Faulkner, K. K. McCully, D. S. Carlson and J. A. MCNamara JR \\ Departments of Physiology and Anatomy, University of Michigan, School of Medicine. \\ Ann Arbor, MI 48109, U.S.A.
}

\begin{abstract}
Summary-The hypothesis was tested that increasing the resting length of the masseter and temporalis muscles by a bite-opening appliance with or without detachment and re-attachment of the masseter would not affect the contractile properties of these muscles. Appliances opened the bite of 10 adult female monkeys $20 \mathrm{~mm}$. Five received the appliance alone (Group A); five received the appliance and in addition the masseter was detached and re-attached (Group ADR). Comparisons were made 48 weeks later. Small bundles of fibres were excised from the masseter and temporalis muscles of experimental animals and from 8 control animals. Isometric and isotonic contractile properties were measured in vitro and fibre classification and fibre areas were determined histochemically. No significant differences were observed within either masseter or temporalis muscles between animals in Groups A and ADR. In both groups, the bundles of fibres from the masseter had prolonged contraction and relaxation times com pared to control masseter muscles but no difference was observed in the percentage of Type II fibres. As detachment and re-attachment had no significant effect on morphological or physiological characteristics, other than those due to lengthening, this procedure may be useful in decreasing the passive tension induced when orthognathic surgery increases muscle length. The significant prolongation of the contractile response of the masseter is similar to the adaptation induced by long-term stimulation at low frequency.
\end{abstract}

\section{INTRODUCTION}

Studies of muscle adaptation have focused mainly on limb muscles but interest has increased regarding the adaptive response of muscles in the craniofacial region to alteration in length. Surgical procedures to correct vertical maxillary dysplasia commonly include inter-positional bone grafts in the maxilla designed to increase lower facial height thus lengthening the masseter, medial pterygoid and temporalis muscles. Similarly, mandibular advancement results in a lengthening of the suprahyoid muscle complex. The amount of skeletal relapse after these surgical procedures has led to the assumption that increased muscle length leads to increased passive tension which ultimately causes skeletal displacement (Carlson et al.. 1982). Although detachment of lengthened muscles has been advocated to alleviate distracting forces, the effect of lengthening and surgical detachment on the function of skeletal muscles is unknown.

In a long-term study of altered facial height using a bite-opening appliance with or without detachment and re-attachment of the masseter (Maxwell et al., 1981), the masseter and temporalis muscles showed no changes in percentage of Type II fibres and minimal changes in cross-sectional area of fibres. Significant adaptations included a decrease in the oxidative capacity of both muscles and an increased number of sarcomeres per fibre in the temporalis muscle. Our purpose was to determine the effects on the contractile properties of the masseter and temporalis muscles of increasing the resting length of these muscles and of increasing the resting length of these muscles with detachment and re-attachment of the masseter. Burke et al. (1971) reported a strong correlation between fibre composition determined histochemically and contractile properties of limb muscles. Based on this correlation and our observations that lengthening, detachment and re-attachment did not produce significant differences in the fibre composition of the muscles of mastication (Maxwell et al., 1981), we hypothesized that introduction of the appliance either with or without detachment and re-attachment of the masseter muscle would not affect the contractile propertics of the masseter or temporalis muscles.

\section{MATERIALS AND METHODS}

Eighteen adult female rhesus monkeys (Macaca mulatta) were quarantined in our animal care facilities. The dentitions indicated that the animals were at least 4.5 years old (Hurme and Van Wagenen, 1961). Their weights ranged from 5.8 to $8.0 \mathrm{~kg}$. For each experimental procedure. the animals were restrained by an intramuscular injection of ketamine and anaesthetized with an intravenous injection of pentobarbital.

Each of 10 experimental monkeys received a biteopening appliance (McNamara, 1977) cast in ticonium and cemented to the maxillary arch. The appliance displaced the mandible infero-posteriorly, opened the bite approximately $20 \mathrm{~mm}$ incisally and 
resulted in a 15 per cent bilateral lengthening of the masseter and temporalis muscles (Carlson, 1977). Animals adjusted to the appliance and appeared to experience no major problems in feeding.

The 5 monkeys in Group A (Appliance) received an appliance only and were subjected to no other experimental manipulations except routine radiological examination. The 5 monkeys in Group ADR (Appliance/Detachment/Re-attachment) had an appliance cemented to the maxillary arch. In addition, the masseter muscles were detached bilaterally and immediately re-attached to the pterygomasseteric sling (Yellich et al., 1981).

Control data were collected on 8 monkeys. Because these animals were used for measurements of skeletal muscle blood flow (White et al., 1981), the animals were killed by overdose of pentabarbital at the termination of the experiments. A biopsy sample was removed from the superficial anterior portion of one masseter and one temporalis muscle. Each biopsy sample included intact fibres that extended from the superficial facial sheath to the intermediate facial sheath. Bundles of intact fibres were isolated from the biopsy sample by micro-surgical techniques using a dissection microscope. Ties were placed around the proximal and distal ends of the intact fibres at the point where they inserted into each facial sheath.

Forty-eight weeks after the appliance had been inserted, small biopsies were removed using a sterile surgical technique. The monkeys were not killed at the termination of these experiments. Biopsies were obtained from the same portion of the masseter and the temporalis muscles as those from control animals, but the fibres were usually cut proximal to the intermediate facial sheath. Consequently, the open biopsies obtained from the experimental animals were shorter than those obtained from the control animals. A tie was placed around each bundle just proximal to the cut end.

Isometric and isotonic contractile properties were measured in vitro on the bundles of fibres including: time to peak twitch tension (TPT), the relaxation time from maximum to half maximum twitch tension (1/2 RT), maximum twitch tension $\left(P_{1}\right)$, the twitch tension to tetanus tension ratio, maximum isometric tetanus tension $\left(\mathbf{P}_{0}\right)$ the force-frequency relationship, the maximum velocity of shortening (Faulkner et al., 1982). Preparations were mounted in a muscle bath containing $80 \mathrm{ml}$ of Krebs bicarbonate saline solution. One end of the muscle preparation was tied to a stationary hook at the bottom of the bath. The other end was tied to an isotonic lever in series with an isometric-force transducer. Preparations were stimulated with platinum field-electrodes set $10 \mathrm{~mm}$ apart. Stimulations was supramaximal unidirectional square pulses of $200 \mu$ s duration. Muscle fibre length was adjusted to an optimal length $\left(\mathrm{L}_{0}\right)$ such that a single stimulus pulse elicited $\mathrm{P}_{\mathrm{r}}$. Muscle preparations were stimulated at $10,20,35,50,80$ and 100 pulses per second $(\mathrm{Hz})$. If isometric tension at $100 \mathrm{~Hz}$ was greater than tension at $80 \mathrm{~Hz}$, stimulation frequency waş increased until $\mathrm{P}_{0}$ was reached.

For measurements of shortening velocity, the isotonic lever was after-loaded with a range of forces from 0.05 to 0.7 of $P_{0}$. The muscles were stimulated for $1 \mathrm{~s}$ at the frequency which resulted in $P_{0}$. The bundles of fibres were allowed to shorten $1 \mathrm{~mm}$ before movement was halted by the lever coming into contact with an isometric force transducer. This was necessary because some preparations showed a decrease in $\mathrm{P}_{0}$ with time. The length of each muscle bundle was measured between the ties with the muscle fibres at $L_{0}$. The velocity of shortening was expressed as $\mathrm{L}_{0}$ per second. Each value for velocity of shortening was plotted against the appropriate relative after-load. The maximum velocity of shortening was estimated from the linear expression of the Hill equation (Hill, 1938).

The length of each muscle bundle was corrected for the damaged, non-functioning region adjacent to the ties (Faulkner et al., 1982). The extent of this damage was estimated by allowing four muscle bundles to shorten maximally with a minimal load of approximately 5 per cent of $P_{0}$. Under these circumstances, intact bundles of diaphragm muscle fibres from rats shortened 49 per cent of $\mathrm{L}_{0}$. Therefore, we assumed that under the same circumstances the undamaged portion of bundles from the masseter and temporalis muscles would shorten the same amount. If a bundle with a $16 \mathrm{~mm}$ fibre length shortened $5.1 \mathrm{~mm}$, the functional fibre length was $5.1 / 0.49=10.4 \mathrm{~mm}$. Using this correction, the mean functional fibre length of masseter muscles was 66 per cent of the measured fibre length and that of the temporalis was 55 per cent. The mean corrected fibre lengths for control and experimental muscles respectively was 10.3 and $5.6 \mathrm{~mm}$ for masseter and 12.9 and $7.8 \mathrm{~mm}$ for the temporalis muscles.

After measurement of contractile properties, the bundle was removed, blotted and weighed. Cross-sectional area was estimated from the mass of the bundle and fibre length assuming a muscle density of 1.06 and a uniform cross-section (Close, 1972). The absolute value of $\mathrm{P}_{0}$ (in newtons) was divided by the cross-sectional area $\left(\mathrm{cm}^{2}\right)$ to normalize tension (N. $\mathrm{cm}^{-2}$ ).

Histochemical analysis of the muscle bundles was performed as reported by Maxwell et al. (1981). A sample of the complete cross-sectional area was cut from the centre portion of each bundle and then quick-frozen in isopentane cooled with dry ice. Sections $14 \mu \mathrm{m}$ thick were cut in a cryostat and then incubated at $\mathrm{pH} 9.4$ for myofibrillar ATPase activity by the method of Chayen, Bitensky and Butcher (1973). Sections were projected, fibre outlines traced and fibres classified on the basis of low or high myofibrillar ATPase activity as either Type I or Type II fibres (Brooke and Kaiser, 1970). Fibre areas were obtained by planimetry and the Type II fibre area was calculated for each bundle.

Significant differences between bundles of fibres from different muscles were tested for by StudentFisher $t$-tests (accepting $p<0.05$ ). Data are presented as means \pm one standard error of the mean (SEM).

\section{RESULTS}

No significant differences were observed between the contractile properties of either the masseter or temporalis muscles obtained from monkeys in Group A and Group ADR (Table 1). Therefore, for all subsequent comparisons with control values, the data 
Table 1. Contractile properties of masseter and temporalis muscles from female rhesus monkeys with bite-opening appliance (Group A) and with an appliance and detachment and re-attachment of the masseter (Group ADR)

\begin{tabular}{lcccc}
\hline & \multicolumn{2}{c}{ Masseter } & \multicolumn{2}{c}{ Temporalis } \\
& Group A & Group ADR & Group A & Group ADR \\
\hline TPT $(\mathrm{ms})$ & $35.2 \pm 2.9$ & $36.0 \pm 1.5$ & $25.1 \pm 0.5$ & $27.3 \pm 3.0$ \\
$1 / 2 \mathrm{RT}(\mathrm{ms})$ & $28.7 \pm 1.6$ & $27.5 \pm 0.3$ & $18.5 \pm 0.8$ & $19.0 \pm 2.1$ \\
$\mathrm{P}_{\mathrm{t}} / \mathrm{P}_{0}$ & $0.19 \pm 0.04$ & $0.24 \pm 0.02$ & $0.18 \pm 0.02$ & $0.20 \pm 0.1$ \\
$\mathrm{P}_{0}\left(\mathrm{~N} . \mathrm{cm}^{-2}\right)$ & $7.2 \pm 1.0$ & $7.0 \pm 1.5$ & $5.6 \pm 1.2$ & $7.7 \pm 2.5$ \\
\hline
\end{tabular}

No significant differences were observed $(p>0.05)$.

from the two groups were pooled (Table 2). The length and cross-sectional areas of bundles of fibres from masseter and temporalis muscles from experimental monkeys were significantly smaller than bundles obtained from control animals. Consequently, the maximal twitch and tetanus tensions were 60-70 per cent lower (Table 2). The normalized $\mathrm{P}_{0}\left(\mathrm{~N} . \mathrm{cm}^{-2}\right)$ of the bundles of muscle fibres from the experimental animals was also significantly less (44-63 per cent) than the value for control animals. The TPT of the masseter and temporalis muscles and the 1/2 RT of the masseter muscle were significantly longer for bundles from experimental animals than for control animals.

The reciprocal of the TPT is a linear function of the percentage of Type II fibres (Fig. 1). The data for the masseter and temporalis muscles from control animals and for the temporalis of experimental animals were on the same regression line. The regression equation for the control data is $1 /$ TPT $=0.032$ $+0.00022 \times$ per cent Type II fibre area. The data for the masseter muscles from animals with an appliance are on a significantly different regression line: $1 / \mathrm{TPT}=0.027+0.00011 \times$ per cent Type II fibre area.

We had expected that the force-frequency relation might provide an accurate estimate of changes in contractile properties. Unfortunately, the variability within groups was so great that no significant differ- ences were observed either between masseter and temporalis muscles in either group or between experimental and control muscles. The pooled data for masseter and for temporalis muscles are presented in Fig. 2.

The data for the uncorrected shortening velocity of individual bundles from masseter and temporalis muscles from control and experimental monkeys are plotted against 1/TPT (Fig. 3). The values obtained after correcting for the damage inflicted by the cutting and tying of fibres are in good agreement with the regression line between maximum velocity of shortening and 1/TPT (Close, 1966).

\section{DISCUSSION}

In a related histochemical study, no differences were found between Groups A and ADR in crosssectional area, fibre length of fibre composition of the masseter or temporalis muscles (Maxwell et al., 1981). We now report no difference between the two experimental groups, Group A and Group ADR, in the contractile properties of the masseter muscle. In animals with a bite-opening appliance, the lack of a significant difference following detachment and re-attachment of the masseter suggests this procedure may be useful in the reduction of passive stretch without altering muscle function when muscle length is increased by orthognathic surgical procedures.

Table 2. Contractile properties of masseter and temporalis muscles from control adult female rhesus monkeys and from adult female rhesus monkeys with a bite opening appliance both with and without detachment and re-attachment of the masseter

\begin{tabular}{lcccc}
\hline & \multicolumn{2}{c}{ Masseter } & \multicolumn{2}{c}{ Temporalis } \\
& Control & Appliance & Control & Appliance \\
\hline $\mathrm{L}_{0}(\mathrm{~mm})$ & $15.9 \pm 1.4$ & $8^{*} 4^{*} \pm 0.4$ & $22.7 \pm 1.4$ & $14.8^{*} \pm 1.1$ \\
Cross-sectiont $\left(\mathrm{mm}^{2}\right)$ & $15.4 \pm 2.6$ & $6.6^{*} \pm 0.6$ & $15.2 \pm 3.4$ & $8.5^{*} \pm 1.9$ \\
TPT $(\mathrm{ms})$ & $25.5 \pm 1.4$ & $35.7^{*} \pm 0.7$ & $22.4 \pm 1.1$ & $25.8^{*} \pm 0.9$ \\
$1 / 2 \mathrm{RT}(\mathrm{ms})$ & $21.9 \pm 1.1$ & $27.7^{*} \pm 1.7$ & $18.4 \pm 1.3$ & $18.7 \pm 0.8$ \\
$\mathrm{P}_{1}(\mathrm{~g})$ & $12.6 \pm 3.4$ & $3.9^{*} \pm 1.4$ & $25.6 \pm 5.5$ & $10.7^{*} \pm 2.7$ \\
$\mathbf{P}_{1 / \mathrm{P}_{0}}$ & $0.18 \pm 0.02$ & $0.19 \pm 0.04$ & $0.21 \pm 0.03$ & $0.19 \pm 0.01$ \\
$\mathrm{P}_{0}\left(\mathrm{~N} . \mathrm{cm}^{-2}\right)$ & $12.7 \pm 1.6$ & $7.1^{*} \pm 1.1$ & $19.3 \pm 3.3$ & $7.2^{*} \pm 0.8$ \\
$\mathrm{~V}_{0}\left(\mathrm{~L}_{0} / \mathrm{s}\right)$ & 7.4 & 6.6 & 9.0 & 9.1 \\
Sample size & 12 & 8 & 11 & 12 \\
\hline
\end{tabular}

\footnotetext{
* Indicates a significant difference compared to the appropriate control muscle $(p<0.05)$.

+ Cross-sectional area $(A)$ in $\mathrm{mm}^{2}$ was determined from the equation $A=$ mass $(\mathrm{mg}) /$ fibre length (mm) $\times 1.06$.
} 


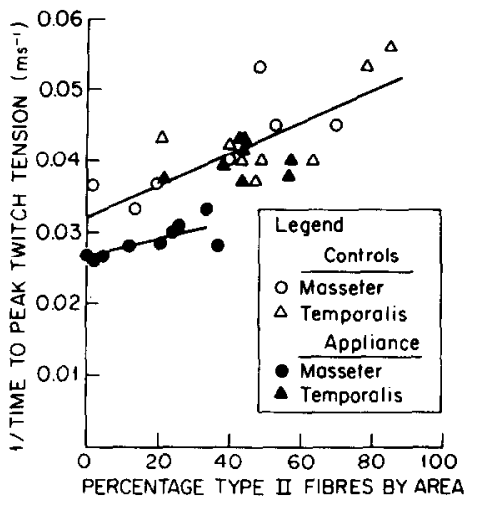

Fig. 1. The relationship between the reciprocal of time to peak twitch tension and the percentage area of Type II fibres in the muscle bundle.

The significant difference in the length and crosssectional area of the bundles from experimental animals compared to control animals resulted from smaller muscle biopsies being taken from experimental animals who would survive than were taken from control animals about to be killed. We have shown in previous experiments on the contractile properties of bundles of fibres that TPT, 1/2 RT, twitch tensionisometric tetanus tension ratio and the normalized $P_{0}$ were not affected by the size of the bundle (Faulkner et al., 1982). Furthermore, TPT and 1/2 RT were not significantly different in bundles with fibres cut at one or both ends compared to bundles of intact fibres.

Normalized $\mathrm{P}_{0}$ of whole muscles is usually between 16 and $30 \mathrm{~N} . \mathrm{cm}^{-2}$ (Close, 1972). Small bundles of intact fibres or of fibre segments range from 10 to $28 \mathrm{~N} . \mathrm{cm}^{-2}$ (Faulkner et al., 1982). Values less than $28-30 \mathrm{~N} . \mathrm{cm}^{-2}$ may result from errors in the estimation of the true cross-sectional area of pennate whole muscles or from damage to fibres in the bundles of fibres. We attribute the significant difference between the normalized $\mathrm{P}_{0}$ of muscles from experimental and control animals to the different techniques used to remove bundles rather than any intrinsic difference in the capacity of bundles to develop tension. Removal of small biopsies can result in significantly more damage to the bundle of fibres ultima-

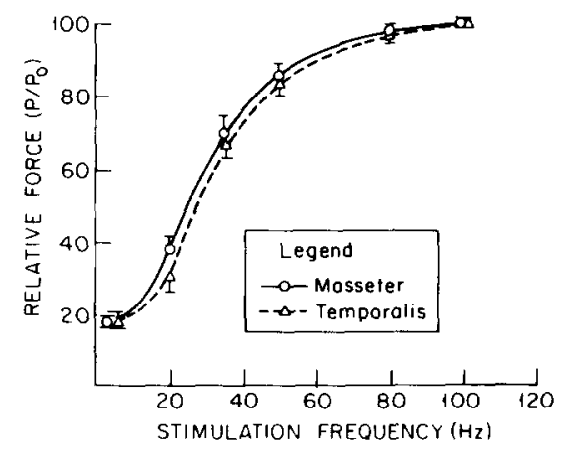

Fig. 2. The relationship between relative force and the frequency of stimulation for masseter and temporalis muscles. No significant differences were observed and data were pooled for experimental and control animals.

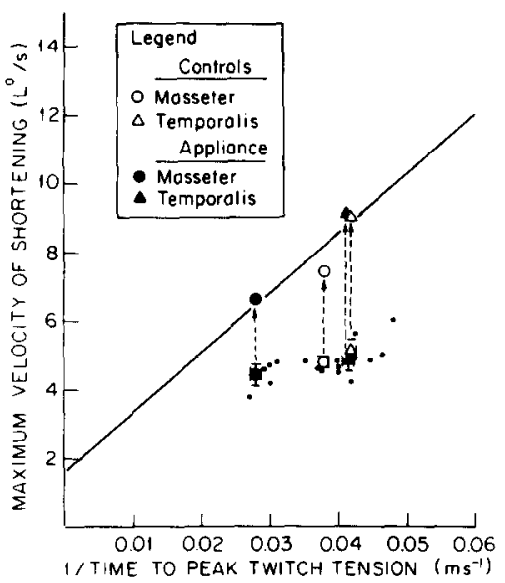

Fig. 3. The relationship between the maximum velocity of shortening and the reciprocal of the time to peak twitch tension. The small dots indicate actual data points. The means of the four groups were corrected (arrows) for the amount of damage adjacent to the ties (see text). The regression line is for data on mammalian limb muscle (Close, 1966).

tely used to measure contractile properties than does the excision of large sections of muscle. Differences in normalized $P_{0}$ in the range reported here does not affect other contractile properties (Faulkner et al., 1982).

We rejected our hypothesis that the appliance would have no effect on the contractile properties. Compared to control muscles, the significant prolongation of the TPT and 1/2RT of the masseter 48 weeks after insertion of an appliance suggests major biochemical adaptations. The biochemical adaptations that would produce prolongation of the contraction time are an increase in the proportions of slow myosin in masseter fibres, a decrease in the rate of calcium uptake by the sarcoplasmic reticulum or some combination of these factors (Salmons and Henriksson, 1982).

The physiological mechanism that triggered the prolongation of the times for contraction and relaxation is not immediately clear. Stretch coupled with immobilization produces significant hypertrophy (Holly et al., 1980; Laurent and Millward, 1980). In our experiment, stretch coupled with activity gave evidence of fibre atrophy rather than hypertrophy (Maxwell et al., (1981). D. S. Carlson and J. A. McNamara (data not presented here) found increased electromyographic activity in the masseter and temporalis muscles during the first few weeks after insertion of the appliance but this gradually reverted to control levels. A month or more of stimulation at low frequency $(10 \mathrm{~Hz})$ is known to induce synthesis of slow myosin and produce a longer contraction time (Salmons and Henriksson, 1981). We have no electromyographic evidence of the long-term changes in recruitment necessary to produce this type of stimulus.

The significant prolongation of the contractile response with no significant change in the proportion of fibres with a high myofibrillar ATPase (Type II fibres) adds further support to the contention of Guth and Samaha (1972) that erroneous interpretations may 
result from histochemical observations. Such a discrepancy is apparent in Fig. 1. For a given percentage of Type II fibres, the TPT of masseter muscles from monkeys with an appliance is significantly longer (lower 1/TPT) than control masseter muscles. The proportions of Type II fibres in the four groups are within the ranges expected from our previous study (Maxwell et al., 1981).

The maximum velocity of shortening has not been reported previously for any of the muscles of mastication. Statistical comparisons of the uncorrected data indicate no significant difference between the maximum velocity of shortening of experimental and control masseter muscles. After a correction was made for damaged sarcomeres near the cut or tied ends (Faulkner et al., 1982) maximum velocities of shortening were 7.4 and $6.6 \mathrm{~L}_{0}$ / $\mathrm{s}$ for the control and experimental masseter muscles and 9.0 and $9.1 \mathrm{~L}_{0} / \mathrm{s}$ for the control and experimental temporalis muscles. The close agreement of the corrected values with the linear relationships between maximum velocity of shortening and 1/TPT reported for whole limb muscles by Close (1966) and for small bundles of intact fibres or fibre segments by Faulkner et al. (1982) suggests that the method of correction is reasonable. The correction of the mean value does not permit statistical treatment of the data. Therefore, data on the maximum velocity of shortening do not provide any insight as to the mechanism by which the TPT and 1/2 RT are lengthened.

Acknowledgements . We thank Carol Brangwyn for her careful handling of the histochemical data, Jody Ungerleider for management of the monkeys used in this analysis, and Timothy P. White for his helpful comments on our manuscript. The research was supported by United States Public Health Service grants DE 04227, DE 05232 and DE 04857 from the National Institute of Dental Research, National Institutes of Health. Bethesda. Md.. U.S.A.

\section{REFERENCES}

Brooke M. H. and Kaiser K. K. 1970. Muscle fiber types: how many and what kind. Archs Neurol. 23, 369-379.

Burke R. E., Levine D. N.. Zajac F. E. III, Tsairis P. and Engel W. K. 1971. Mammalian motor units: physiological-histochcmical correlates in three types in cat gastrocnemius. Science 174, 709-712.

Carlson D. S. 1977. Condylar translation and the function of the superficial masseter muscle in the rhesus monkey (M. mulatta). Am. J. phis. Anthrop. 47, 53-63.

Carlson D. S., Ellis E. III, Schneiderman E. D. and Unger- leider J. C. 1982. Experimental models of surgical intervention in the growing face: Cephalometric analysis of facial growth and relapse. In: Effect of Surgical Intervention on Craniofacial Growth (Edited by McNamara J. A Jr, Carlson D. S. and Ribbens K.) Monograph No. 12. Craniofacial Growth Series, Center for Human Growth and Development, The University of Michigan, Ann Arbor, pp. 11-72.

Chayen J., Bitensky L. and Butcher R. G. 1973. Part I Enzyme Histochemistry-Adenosine Triphosphatases. Practical Histochemistry, pp. 122-124. Wiley, New York.

Close R. 1966. Dynamic properties of fast and slow skeletal muscles of mammals. In: Exploratory Concepts in Muscular Dvstrophl and Related disorders (Edited by Millorat A. J.) pp. 142-150. Exerpta Medica Foundation. New York.

Close R. I. 1972. Dynamic properties of muscle. Physiol Ret. 52, 129-197.

Faulkner J. A., Claflin D. R., McCully K. K. and Jones D. A. 1982. Contractile properties of bundles of fiber segments from skeletal muscles. Am. J. Physiol. Cell Physiol. 12. C66-C73.

Guth L and Samaha F. J. 1972. Erroneous interpretations which may result from application of the "myofibrillar ATPase" histochemical procedure 10 developing muscle. Expl Neurol. 34, 465-475.

Hill A. V. 1938. The heat of shortening and the dynamic constants of muscle. Proc. R. Soc. Lond. Ser. B 126, 136-195.

Holly R. G., Barnett J. G., Ashmore C. F., Taylor R. G. and Mole P. A. 1980. Stretch-induced growth in chicken wing muscles: A new model of stretch hypertrophy. Am. J. Phisiol. 7, C62-C71.

Hurme V. O. and Van Wagenen E. G. 1961. Basic data on the permanent teeth in the rehesus monkey. Proc. Am phil. Soc. 105, 105-140.

Laurent G. J. and Milward D. J. 1980. Protein turnover during skeletal muscle hypertrophy. Fed. Pros. 39, $42-47$.

Maxwell L. C., Carlson D. S., McNamara J. A. and Faulkner J. A. 1981. Adaptation of the masseter and temporalis muscles following alteration in length. with or without surgical detachment. Anat. Rec: 200, 127-137.

McNamara J. A. Jr. 1977. An experimental study of increased vertical dimension in the growing face. $4 \mathrm{~m} . \mathrm{J}$. Orthod. 71, 382395 .

Salmons S. and Henriksson J. 1981. The adaptive response of skeletal muscle to increased use. Muscle Nerte 4 , $94-105$.

White T. P.. Pachtman S.. Villanacci J. F.. Faulkner J. A. and McNamara J. A, 1979. Blood flow of masticatory muscles of rhesus monkeys. Physiology 22, 132.

Yellich G. M.. McNamara J. A. Jr and Ungerleider J. S. 1981. Muscular and mandibular adaptation after lengthening. detachment and reattachment of the masseter muscle. J. oral Sura. 39, 656665. 